\section{Computing Global Combine Operations in the Multiport Postal Model}

\author{
Amotz Bar-Noy, Jehoshua Bruck, Ching-Tien Ho, \\ Shlomo Kipnis, and Baruch Schieber
}

\begin{abstract}
Consider a message-passing system of $\boldsymbol{n}$ processors, in which each processor holds one piece of data initially. The goal is to compute an associative and commutative reduction function on the $n$ pieces of data and to make the result known to all the $n$ processors. This operation is frequently used in many message-passing systems and is typically referred to as global combine, census computation, or gossiping. This paper explores the problem of global combine in the multiport postal model. This model is characterized by three parameters: $n$-the number of processors, $k$-the number of ports per processor, and $\lambda$-the communication latency. In this model, in every round $r$, each processor can send $\boldsymbol{k}$ distinct messages to $\boldsymbol{k}$ other processors, and it can receive $\boldsymbol{k}$ messages that were sent from $k$ other processors $\lambda-1$ rounds earlier. This paper provides an optimal algorithm for the global combine problem that requires the least number of communication rounds and minimizes the time spent by any processor in sending and receiving messages.
\end{abstract}

Index Terms-Census computation, distributed systems, global combine, gossiping, message-passing systems, multiple ports, parallel computers, postal model.

\section{INTRODUCTION}

This paper explores the global combine operation in the multiport postal model for message-passing systems. In this model, the system consists of $n$ processors, each processor has $k$ communication ports, and the communication latency is $\lambda$. We denote such a messagepassing system by $\mathcal{M P S}(n, k, \lambda)$, and we assume that the $n$ processors are labeled from 0 through $n-1$. In the global combine operation, each processor $i$ initially holds a data item $d_{i}$. Given a commutative and associative combining operator " $\oplus$," the goal is to compute $D=d_{0} \oplus d_{1} \oplus \cdots \oplus d_{n-1}$ and to place the result $D$ in all the $n$ processors. Examples of such combining operators are MAX, MIN, (logical or bitwise) AND, OR, XOR, integer addition, and integer multiplication.

The global combine operation is frequently used in many applications for message-passing systems (see [20]). Several collective communication libraries, such as Express [19] by Parasoft and the External User Interface (EUI) [2], [3] of the Scalable POWERparallel System (SP-1) by IBM, provide primitives for computing a global combine operation. This operation has also been included as part of the collective communication routines in the Message-Passing Interface (MPI) standard proposal [18]. (This operation is called mpi_allreduce in MPI.) In the literature, this operation is also referred to as census computation (e.g., [1], [8]) or gossiping [21].

In the multiport postal model, two integer parameters, $k \geq 1$ and $\lambda \geq 1$, are used to model the communication characteristics of a message-passing system $\mathcal{M P S}(n, k, \lambda)$. We assume that each of the

Manuscript received June 8, 1994; revised Oct. 19, 1994.

A. Bar-Noy is with the EE Department, Tel Aviv University, Ramat Aviv, Tel Aviv, 69978, Israel; and IBM T.J. Watson Research Center, Yorktown Heights, NY 10598; e-mail: amotz@eng.tau.ac.il.

B. Schieber is with the IBM T.J. Watson Research Center, Yorktown Heights, NY 10598; e-mail: sbar@watson.ibm.com.

J. Bruck is with the California Institute of Technology, mail code 116-81, Pasadena, CA 91125; e-mail: bruck@ systems.caltech.edu.

C.T. Ho is with the IBM Almaden Research Center, 650 Harty Road, San Jose, CA 95120; e-mail: ho@almaden.ibm.com.

$S$. Kipnis is with the IBM Israel Science and Technology Center, MATAM-Advanced Technology Center, Haifa, Israel 31905; e-mail:

kipnis@haifasc3.vnet.ibm.com.

IEEECS Log Number D95022. $n$ processors has "effectively" $k$ distinct input ports and $k$ distinct output ports, and we assume that communicating a data item between any pair of processors exhibits a latency of $\lambda$. In other words, we assume that in each communication round $r$, every processor can send $k$ distinct messages to $k$ other processors, and in the same round $r$ each processor can receive $k$ distinct messages that were sent out from $k$ other processors $\lambda-1$ rounds earlier (i.e., in round $r-\lambda+1$, provided that $r-\lambda+1 \geq 1$ ). It is assumed that all processors know the values of $n, k$, and $\lambda$.

The multiport postal model generalizes existing models in two ways:

1) by addressing multiport capabilities of several systems $(k>1)$, and

2) by addressing communication latencies of systems $(\lambda>1)$. Although we do not know any existing machines that exhibit the multiport postal model with $k>1$ and $\lambda>1$, there have been many machines exhibit either the multiport model or the postal model. This is the first attempt to unify the algorithm design and implementation for both models, by considering algorithm for the more general model. Although we consider the global combine operation only in this paper, the same techniques can be easily applied to algorithms for all-to-all broadcast under the same model.

In the first way, the multiport model generalizes the one-port model that has been widely investigated. There are two prevailing variants of the one-port model: unidirectional communication and bidirectional communication. In unidirectional communication, a processor can either send or receive a message in any given round (see [21]). In bidirectional communication, distinct pairs of processors can exchange messages in any given round (see [21], [22], [26]). Recently, a third variant was explored, in which in any given round, each processor can send a message to one processor and receive a message from another processor (see [7], [8], [11], [12]). However, there are examples of parallel systems with $k$-port capabilities for $k>1$, such as the Ncube/2 [25] and the CM-2 (where $k$ is the dimension of the hypercube in both machines).

In the second way, the postal model, which was recently introduced by Bar-Noy and Kipnis [5], [6] addresses emerging trends in modern distributed-memory parallel computers and high-speed communication networks. (A related model, the LogP model [17], was also proposed recently to address similar goals.) Systems that are well modeled by the postal model include parallel computers like TMC's CM-5 [24] and IBM's SP-2 [28], and communication networks such as PARIS [14] and AURORA [15]. Bruck et al. has confirmed experimentally in [10] that a properly tuned broadcast based on the postal model on the Delta system can improve the best known implementation by more than $20 \%$. Culler et al. also confirmed experimentally in [16] that LogP model is helpful in the development of the fast parallel sorting algorithms on the CM-5.

Two complexity measures are frequently used in evaluating communication algorithms. The first measure, denoted by $C_{1}$, is the number of communication rounds required by an algorithm. Notice that in each round, the messages sent and received by each processor can be of arbitrary sizes. Let $s_{i}$ be the size of the largest message sent or received by any processor in round $i$. The second measure, denoted by $C_{2}$, is the sum of the $s_{i} \mathrm{~s}$ over all the rounds. Note that $C_{2}$ gives an upper bound on the number of data items sent or received per processor. Hence, the communication time complexity of an algorithm can be modeled as $C_{1} t_{s}+C_{2} t_{c}$, where $t_{s}$ is the communication start-up time and $t_{c}$ is the per-data-item communication time. 
Many algorithms for the global combine operation have appeared in the literature. Most of these algorithms are based on the one-port no-latency model $(k=\lambda=1)$ and are restricted to certain topologies, such as meshes [4] or hypercubes [20], [27]. A naive way to compute the global combining operation is to solve the concatenation problem (see [12] for an optimal solution). However, since in this solution $C_{2}$ is very large (order of $n$ ), we rule out such an approach.

In the general $\mathcal{M P S}(n, k, \lambda)$ model, optimal solutions for the global combine problem were known only for specific values of the parameters, $n, k$, and $\lambda$. For example, for the case of $k=\lambda=1$, a known solution first reduces the number of data pieces to be a power of 2 , then applies a butterfly combining algorithm on the reduced data, and finally sends the reduction result to the remaining processors. This solution is optimal in both $C_{1}$ and $C_{2}$ only when $n$ is a power of 2 . For the same parameters, an optimal solution for any value of $n$ was recently developed in [8]. This solution is based on the binary representation of $n$. Lately, an optimal solution for $k=1$, for any integer $\lambda \geq 1$, but only for values of $n$ of certain form, was presented in [23]. It should be noted that the solution of [23] can be extended to any value of $n$ (still with $k=1$ ). This extension results in an algorithm that is within an additive term of $2 \lambda-1$ of optimal. More recently, two new optimal solutions for the global combine problem in restricted models have appeared: 1) a solution for $k=1$ and for any integers $\lambda \geq 1$ and $n \geq 1$ was presented in [9], and 2) a solution for $\lambda=1$ and for any integers $k \geq 1$ and $n \geq 1$ appeared in [13]. Finally, it should be noted that if the combining operator " $\oplus$ " satisfies $a \oplus a=a$ for all possible $a$ (such as MAX and MIN), then solutions for "nice" numbers can be extended to an optimal solution for any value of $n$ [8]. However, many operators do not have this property (e.g., Exclusive Or, integer addition, and integer multiplication).

In this paper, we provide for the first time an optimal algorithm (with respect to both $C_{1}$ and $C_{2}$ ) for the global combine problem for any commutative and associative operator and for any integers $n \geq 1$, $k \geq 1$ and $\lambda \geq 1$.

\section{PRELIMINARIES}

In this section, we provide definitions that are used by the general algorithm in Section III. We also provide the lower bound for the global combine problem.

\section{A. Sequence Representation of Integers}

We first define a sequence of generalized Fibonacci numbers that is indexed by $k$ and $\lambda$. Given integers $k \geq 1$ and $\lambda \geq 1$, the function $F($.$) is defined from the nonnegative integers into the positive inte-$ gers as follows:

$$
F(r)= \begin{cases}1 & \text { if } 0 \leq r \leq \lambda-1, \\ F(r-1)+k F(r-\lambda) & \text { if } \lambda \leq r .\end{cases}
$$

For clarity, the two parameters $k$ and $\lambda$, which are fixed throughout the paper, are omitted from the function $F$. For example, for $\lambda=1$, we have $F(r)=(k+1)^{r}$, and for $k=1$ and $\lambda=2$ the value of $F(r)$ is the Fibonacci number whose index is $r$.

In Section III, we show that the variable $r$ in $F(r)$ corresponds to the round number in the algorithm. Thus, if the number of processors $n>1$ is of the form $n=F(m)$, for some integer $m$, then the global combine algorithm finishes optimally in $m$ rounds. However, it is more complicated to give an optimal algorithm that takes $m$ rounds for $n>1$ when $F(m-1)<n<F(m)$. In order to do so, we need to introduce a sequence of deficiency parameters $\varepsilon=\left(\varepsilon_{0}, \varepsilon_{1}, \ldots, \varepsilon_{m-\lambda}\right)$, where the $\varepsilon_{i}$ s are integers.
Formally, let $\varepsilon=\left(\varepsilon_{0}, \varepsilon_{1}, \ldots, \varepsilon_{m-\lambda}\right)$ be a sequence in which $0 \leq \varepsilon_{j} \leq k$, for all $0 \leq j \leq m-\lambda$. Define the following recurrence:

$$
F_{\varepsilon}(r)= \begin{cases}1 & \text { if } 0 \leq r \leq \lambda-1, \\ F_{\varepsilon}(r-1)+k F_{\varepsilon}(r-\lambda)-\varepsilon_{r-\lambda} & \text { if } \lambda \leq r \leq m\end{cases}
$$

Note that the recurrence $F($.$) is identical to F_{\varepsilon}($.$) , for \varepsilon=(0, \ldots, 0)$ and $r \leq m$. We denote this recurrence by $F_{0}($.$) .$

Assume, throughout the paper, that $F_{0}(m-1)<n \leq F_{0}(m)$. In the following, we show that there exists a sequence $\varepsilon=\left(\varepsilon_{0}, \varepsilon_{1}, \ldots, \varepsilon_{m-\lambda}\right)$, in which $0 \leq \varepsilon_{j} \leq k$, for all $0 \leq j \leq m-\lambda$, such that $n=F_{\mathrm{e}}(m)$. First, we need two lemmas.

LEMMA 1. For every $\varepsilon=\left(\varepsilon_{0}, \varepsilon_{j}, \ldots, \varepsilon_{m-\lambda}\right)$, where $0 \leq \varepsilon_{j} \leq \mathrm{k}$, for all $0 \leq j \leq m-\lambda$, we have

$$
F_{\varepsilon}(m)=F_{0}(m)-\sum_{j=0}^{m-\lambda} \varepsilon_{j} F_{0}(m-\lambda-j) .
$$

Proof. The proof is by induction on $m \geq 0$. To obtain the induction basis, let $0 \leq m \leq \lambda-1$. By our definition $F_{\varepsilon}(m)=F_{0}(m)=1$, and the claim is trivially true since there are no summands in the summation. Assume now that the claim is true for all $0 \leq j \leq m-1$. By the induction hypothesis we get the following two equations:

$$
\begin{aligned}
F_{\varepsilon}(m-\lambda)= & F_{0}(m-\lambda)-\sum_{j=0}^{m-\lambda-\lambda} \varepsilon_{j} F_{0}(m-\lambda-\lambda-j) \\
F_{\varepsilon}(m-1)= & F_{0}(m-1)-\sum_{j=0}^{m-1-\lambda} \varepsilon_{j} F_{0}(m-1-\lambda-j) \\
= & F_{0}(m-1)-\sum_{j=0}^{m-\lambda-\lambda} \varepsilon_{j} F_{0}(m-1-\lambda-j) \\
& -\sum_{j=0}^{\lambda-2} \varepsilon_{m-1-\lambda-j} F_{0}(j) .
\end{aligned}
$$

Notice that we partitioned the sum in the second equation into two, and changed the running index of the second sum. By our definition

$$
\begin{aligned}
F_{\varepsilon}(m)= & F_{\varepsilon}(m-1)+k F_{\varepsilon}(m-\lambda)-\varepsilon_{m-\lambda} \\
= & F_{0}(m-1)+k F_{0}(m-\lambda)-\sum_{j=0}^{m-2 \lambda} \varepsilon_{j} F_{0}(m-1-\lambda-j) \\
& -\sum_{j=0}^{m-2 \lambda} k \varepsilon_{j} F_{0}(m-\lambda-\lambda-j)-\sum_{j=0}^{\lambda-2} \varepsilon_{m-1-\lambda-j} F_{0}(j)-\varepsilon_{m-\lambda} \\
= & F_{0}(m)-\sum_{j=0}^{m-2 \lambda} \varepsilon_{j} F_{0}(m-\lambda-j)-\sum_{j=0}^{\lambda-2} \varepsilon_{m-1-\lambda-j} F_{0}(j)-\varepsilon_{m-\lambda} .
\end{aligned}
$$

The first equality follows from the definition of $F_{\varepsilon}($.$) . The second$ equality follows from the induction hypothesis and by rearranging operands. The third equality follows from the definition of $F_{0}($.$) .$ Now, observe that $F_{0}(j)=F_{0}(j+1)=1$, for $0 \leq j \leq \lambda-2$, and thus

$$
\begin{aligned}
F_{\varepsilon}(m)= & F_{0}(m)-\sum_{j=0}^{m-2 \lambda} \varepsilon_{j} F_{0}(m-\lambda-j) \\
& -\sum_{j=0}^{\lambda-2} \varepsilon_{m-1-\lambda-j} F_{0}(j+1)-\varepsilon_{m-\lambda} F_{0}(0) .
\end{aligned}
$$


Finally, by changing the running index in the second summation and by combining all the terms under one summation, we get that $F_{\varepsilon}(m)=F_{0}(m)-\sum_{j=0}^{m-\lambda} \varepsilon_{j} F_{0}(m-\lambda-j)$.

Recall that $F_{0}(m-1)<n \leq F_{0}(m)$, and let $\alpha=F_{0}(m)-n$.

LEMMA 2. There exists $\varepsilon=\left(\varepsilon_{0}, \varepsilon_{1}, \ldots, \varepsilon_{m-\lambda}\right)$, in which $0 \leq \varepsilon_{j} \leq k$, for all $0 \leq j \leq m-\lambda$, such that $\alpha=\sum_{j=0}^{m-\lambda} \varepsilon_{j} F_{0}(m-\lambda-j)$.

PROOF. If $\alpha=0$, then by letting $\varepsilon_{j}=0$ for all $j$ we are done. Suppose that $\alpha>0$. By definition we get that $\alpha<F_{0}(m)-F_{0}(m-1)$ $=k F_{0}(m-\lambda)$. Therefore, there exists $0 \leq \varepsilon_{0}<k$ and $\alpha^{\prime}<F_{0}(m-\lambda)$ such that $\alpha=\varepsilon_{0} F_{0}(m-\lambda)+\alpha^{\prime}$. If $\alpha^{\prime}=0$, then we are done. Otherwise, there exists some $x, 0<x \leq m-2 \lambda+1$, such that $F_{0}(m-\lambda-x) \leq \alpha^{\prime}<F_{0}(m-\lambda+1-x)$. Set

$$
\varepsilon_{x}=\left\lfloor\frac{\alpha^{\prime}}{F_{0}(m-\lambda-x)}\right\rfloor,
$$

and let $a^{\prime \prime}=\alpha^{\prime}-\varepsilon_{x} F_{0}(m-\lambda-x)$. It follows that $\alpha^{\prime \prime}<F_{0}(m-\lambda-x)$. We repeat the above process for $\alpha^{\prime \prime}$ as was done for $\alpha^{\prime}$. Since $0 \leq \alpha^{\prime \prime}<\alpha^{\prime}$, it follows that eventually the remainder will be zero and the proof is completed.

Note that for $\lambda>1$ it is enough to set $\varepsilon_{x}=1$ instead of $\varepsilon_{x}=\left\lfloor\frac{\alpha^{\prime}}{F_{0}(m-\lambda-x)}\right\rfloor$. This is true since in this case $\alpha^{\prime \prime}<F_{0}(m-\lambda+1-x)-F_{0}(m-\lambda-x)=k F_{0}(m-\lambda+1-x-\lambda)<k F_{0}(m-\lambda-x)$.

The above lemmas imply the following theorem. Note that for a given $n$, the sequence $\varepsilon$ for which $n=F_{\varepsilon}(m)$ is not necessarily unique.

THEOREM 3. For any $n$, such that $F_{0}(m-1)<n \leq F_{0}(m)$, there exists a sequence $\varepsilon=\left(\varepsilon_{0}, \varepsilon_{1}, \ldots, \varepsilon_{m-\lambda}\right)$, in which $0 \leq \varepsilon_{j} \leq k$, for all $0 \leq j$ $\leq m-\lambda$, such that $n=F_{\varepsilon}(m)$.

\section{B. The Lower Bound}

Given the implicit integer parameters $k \geq 1$ and $\lambda \geq 1$, we define $f(n)=\min _{r}\{F(r) \geq n\}$. Notice that $F(f(n)-1)<n \leq F(f(n))$. We now show that for any $n>1$, any algorithm for the global combine problem in $\mathcal{M P S}(n, k, \lambda)$ requires at least $C_{1}=f(n)$ rounds and communicates at least $C_{2}=f(n)$ data items per processor.

These bounds are derived by reducing the broadcasting problem to the problem of computing a global combine operation. This can be done by assuming that the broadcast source has a data item while all other processors have identity element $I$ as their data items. Thus, the lower bound for the broadcast problem in $\mathcal{M P S}(n, k, \lambda)$ implies the lower bound for the global combine operation.

Now notice that the recursive definition of $F(r)$ is identical to the recursive definition describing the maximum number of processors that can receive the data item of the source after $r$ rounds in $\mathcal{M P S}(n, k, \lambda)$. Thus, the lower bound for broadcast (and also for global combine) in $\mathcal{M P S}(n, k, \lambda)$ is $C_{1}=C_{2}=f(n)$.

\section{THE ALgORITHM}

In this section, we present an optimal algorithm for the global combine operation in $\mathcal{M P S}(n, k, \lambda)$. The basic idea of the algorithm is to treat $n$ as $F_{\varepsilon}(m)$, where $F_{\varepsilon}(m)$ is recursively defined in Section II.
Using this representation, one can start with the value of 1 and get to the value of $n$ in $m$ rounds. During each round $r$, where $\lambda \leq r \leq m$, a processor receives $k$ values from $k$ other processors, each such value being a partial reduction result of either $F_{\varepsilon}(r-\lambda)$ items or $F_{\varepsilon}(r-\lambda)-1$ items, that were sent out during round $r-\lambda+1$.

Let $I$ be the identity data item with respect to $\oplus$. (Note that the existence of the identity data item is assumed only for the purpose of describing the algorithm. In practice, this is not needed.) In the following, the arithmetic for all the processor IDs and the subscripts for the data items is done in modulo $n$. Each processor $i$ maintains the $\varepsilon$ sequence defined by $n, k$, and $\lambda$, another sequence of $m+1$ elements: $c_{i}^{0}, c_{i}^{1}, \ldots, c_{i}^{m}$, and two local variables: $S_{i}$ and $T_{i}$. For the sake of clarity, we denote the values of $S_{i}$ and $T_{i}$ after round $r$ by $S_{i}^{r}$ and $T_{i}^{r}$, respectively. The contents of these variables are as follows.

- $c_{i}^{r}$ : denotes the number of data items on which the reduction function was already performed after round $r$. (The partial reduction result will be stored in $T_{i}^{r}$ ) Initially, $c_{i}^{0}=1$.

- $S_{i}^{r}$ : holds the reduction result of $c_{i}^{r}-1$ consecutive data items (cyclically) starting with $d_{i+1}$, i.e.,

$$
S_{i}^{r}=d_{i+1} \oplus d_{1+2} \oplus \cdots \oplus d_{i+c_{i}^{r}-1} .
$$

Initially, $S_{i}^{0}=I$.

- $T_{i}^{r}$ : holds the reduction result of $c_{i}^{r}$ consecutive data items (cyclically) starting with $d_{i}$, i.e.,

$$
T_{i}^{r}=d_{i} \oplus d_{i+1} \oplus \cdots \oplus d_{i+c_{i}^{r}-1} \cdots
$$

Initially, $T_{i}^{0}=d_{i}$.

Before the algorithm starts, each processor computes the sequence $\varepsilon=\left(\varepsilon_{0}, \varepsilon_{1}, \ldots, \varepsilon_{m-\lambda}\right)$ in which $0 \leq \varepsilon_{j} \leq k$ for all $0 \leq j \leq m-\lambda$ and such that $n=F_{\varepsilon}(m)$. (This computation can be done following the proof of Lemma 2.) Next, each processor $i$ sets $c_{i}^{j}=F_{\varepsilon}(j)$ for all $0 \leq j \leq m$.

Algorithm. The algorithm consists of $m$ rounds. The following is the description of the algorithm performed by processor $i$ in round $r$, for $1 \leq r \leq m$.

1) Perform the following $k$ sends and $k$ receives concurrently.

- If $r \leq m-\lambda+1$, send $T_{i}^{r-1}$ to the following set of $k-\varepsilon_{r-1}$ processors: $\left\{i-c_{i}^{r+\lambda-2}-(x-1) c_{i}^{r-1}\right.$ : for $\left.1 \leq x \leq k-\varepsilon_{r-1}\right\}$.

- If $r \leq m-\lambda+1$, send $S_{i}^{r-1}$ to the following set of $\varepsilon_{r-1}$ processors:

$$
\left\{i-c_{i}^{r+\lambda-2}-(x-1) c_{i}^{r-1}+x-\left(k-\varepsilon_{r-1}\right):\right.
$$

for $\left.k-\varepsilon_{r-1}<x \leq k\right\}$.

- If $r \geq \lambda$, receive the $k$ data items that were sent out to processor $i$ during round $r-\lambda+1$. Denote these $k$ data items by $V_{1}, V_{2}, \ldots, V_{k}$.

2) If $1 \leq r \leq \lambda-1$, then $S_{i}^{r}=S_{i}^{r-1}$ and $T_{i}^{r}=T_{i}^{r-1}$.

3) If $\lambda \leq r \leq m$, compute the following:

- $S_{i}^{r}=S_{i}^{r-1} \oplus V_{1} \oplus V_{2} \oplus \cdots \oplus V_{k}$;

- $T_{i}^{r}=T_{i}^{r-1} \oplus V_{1} \oplus V_{2} \oplus \cdots \oplus V_{k}$.

The final result of the global combine operation over all the $n$ data items is obtained for processor $i$ in the variable $T_{i}$ after round $m$ (that is, in $T_{i}^{m}$ ). 
We now prove the correctness of the algorithm

THEOREM 4. In the above algorithm $T_{i}^{m}=d_{i} \oplus d_{i+1} \oplus \cdots \oplus d_{i-1}$.

PROOF. The proof is by induction on round $r$, for $1 \leq r \leq m$. The induction hypothesis is that after round $r$, where $1 \leq r \leq m$, the following two properties hold for every $0 \leq i \leq n-1$.

1) $S_{i}^{r}=d_{i+1} \oplus d_{i+2} \oplus \cdots \oplus d_{i+c_{i}^{r}-1}$.

2) $T_{i}^{r}=d_{i} \oplus S_{i}^{r}$.

For the basis, consider $1 \leq r \leq \lambda-1$. We have $S_{i}^{r}=I$ and $T_{i}^{r}=d_{i}$, for all $0 \leq r \leq \lambda-1$. Thus, the two properties hold.

Assume, for the sake of the induction hypothesis, that the two properties hold right before round $r$, for some $\lambda \leq r \leq m$. We now wish to show that they still hold after round $r$. Consider Property 1. Assume without loss of generality that $V_{1}, V_{2}, \ldots, V_{k}$ are ordered according to the ascending order (modulo $n$ ) of the sending processor IDs starting from $i$. For convenience, let $y=k-\varepsilon_{r-\lambda}$. From the algorithm, for $1 \leq x \leq y$, we have that $V_{x}=T_{j(x)}^{r-\lambda}$ where $j(x)=i+c_{i}^{r-1}+(x-1) c_{i}^{r-\lambda}$ and for $y<x \leq k$, we have that $V_{x}=S_{j(x)}^{r-\lambda}$ where $j(x)=i+c_{i}^{r-1}+(x-1) c_{i}^{r-\lambda}-(x-y)$. Note that a value received from processor $j(x)$, where $1 \leq x \leq y$, during round $r$ was the partial reduction result of $c_{j(x)}^{r-\lambda}=c_{i}^{r-\lambda}$ consecutive elements starting from processor $j(x)$. Also, a value received from processor $j(x)$, where $y<x \leq k$, during round $r$ was the partial reduction result of $c_{j(x)}^{r-\lambda}-1=c_{i}^{r-\lambda}-1$ consecutive elements starting from processor $j(x)+1$. Thus, all we need to prove are two things: 1) the "last" element covered by $S_{i}^{r-1}$ (and thus also by $T_{i}^{r-1}$ ) is followed by the "first" element covered by $V_{1} ; 2$ ) the "last" element covered by $V_{x}$ is followed by the "first" element covered by $V_{x+1}$, for $1 \leq x<k$.

To prove 1 ), it follows from the definitions that $(j(1)-i) \bmod$ $n=c_{i}^{r-1}$ if $y>0$, and is $c_{i}^{r-1}-1$ if $y=0$. To prove 2), one verifies that $(j(x+1)-j(x)) \bmod n=c_{i}^{r-\lambda}$ for $1 \leq x<y$ and $(j(x+1)-j(x))$ $\bmod n=c_{i}^{r-\lambda}-1$ for $y \leq x \leq k-1$. Thus, Property 1 holds.

Similarly, it can be seen that Property 2 also holds from the induction. Thus, $T_{i}^{m}$ is the reduction result of $c_{i}^{m}=F_{\varepsilon}(m)=n$ consecutive elements starting from item $d_{i}$.

The next complexity statement follows from the description of the algorithm.

THEOREM 5. The algorithm takes $C_{1}=m=f(n)$ rounds and communicates $C_{2}=m=f(n)$ data items.

\section{AN EXAMPLE}

In this section, we elaborate on a concrete example for the integer addition operator. Let $n=9, k=2$, and $\lambda=3$. From the definition of the function $F($.) it follows that $F(0)=F(1)=F(2)=1, F(3)=3$, $F(4)=5, F(5)=7$, and $F(6)=13$. Therefore, the number of rounds in the algorithm is $m=f(n)=6$. Since $F(6)=13$, it follows that $\alpha=F(m)-n=4$. The constructive proof of Lemma 2 implies that $\varepsilon=(1,1,0,0)$. However, for this example we choose another valid value, $\varepsilon=(1,0,0,1)$, i.e., $\varepsilon_{0}=1, \varepsilon_{1}=0, \varepsilon_{2}=0$, and $\varepsilon_{3}=1$. From the definition of $F_{\varepsilon}($.$) it follows that c_{i}^{0}=F_{\varepsilon}(0)=1, c_{i}^{1}=F_{\varepsilon}(1)$
$=1, c_{i}^{2}=F_{\varepsilon}(2)=1, c_{i}^{3}=F_{\varepsilon}(3)=2, c_{i}^{4}=F_{\varepsilon}(4)=4, c_{i}^{5}=F_{\varepsilon}(5)=6$, and $c_{i}^{6}=F_{\varepsilon}(6)=9$.

Suppose now that processor $i$ 's input is $2^{i}$, for $0 \leq i \leq 8$. Let $T^{j}=\left(T_{0}^{j}, T_{1}^{j}, \ldots, T_{8}^{j}\right)$ and $S^{j}=\left(S_{0}^{j}, S_{1}^{j}, \ldots, S_{8}^{j}\right)$. Initially,

$$
\begin{aligned}
& T^{0}=(1,2,4,8,16,32,64,128,256) \\
& S^{0}=(0,0,0,0,0,0,0,0,0) .
\end{aligned}
$$

We now follow the run of the algorithm round after round.

Round 1. Processor $i$ sends $T_{i}^{0}$ to processor $i-1$ and $S_{i}^{0}$ to processor $i-2$. Processor $i$ receives no messages. Therefore,

$$
\begin{aligned}
& T^{1}=(1,2,4,8,16,32,64,128,256) \\
& S^{1}=(0,0,0,0,0,0,0,0,0) .
\end{aligned}
$$

Round 2. Processor $i$ sends $T_{i}^{1}$ to processors $i-2$ and $i-3$. Processor $i$ receives no messages. Therefore,

$$
\begin{aligned}
& T^{2}=(1,2,4,8,16,32,64,128,256) \\
& S^{2}=(0,0,0,0,0,0,0,0,0) .
\end{aligned}
$$

Round 3. Processor $i$ sends $T_{i}^{2}$ to processors $i-4$ and $i-5$. Processor $i$ receives $T_{i+1}^{0}$ and $S_{i+2}^{0}$. Therefore,

$$
\begin{aligned}
& T^{3}=(3,6,12,24,48,96,192,384,257) \\
& S^{3}=(2,4,8,16,32,64,128,256,1) .
\end{aligned}
$$

Round 4. Processor $i$ sends $T_{i}^{3}$ to processors $i-6$ and $S_{i}^{3}$ to processor $i-7$. Processor $i$ receives $T_{i+2}^{\mathrm{l}}$ and $T_{i+3}^{\mathrm{l}}$. Therefore,

$$
\begin{aligned}
& T^{4}=(15,30,60,120,240,480,449,387,263) \\
& S^{4}=(14,28,56,112,224,448,385,259,7) .
\end{aligned}
$$

Round 5. Processor $i$ sends no messages. Processor $i$ receives $T_{i+4}^{2}$ and $T_{i+5}^{2}$. Therefore,

$$
\begin{aligned}
& T^{5}=(63,126,252,504,497,483,455,399,287) \\
& S^{5}=(62,124,248,496,483,451,391,271,31) .
\end{aligned}
$$

Round 6. Processor $i$ sends no messages. Processor $i$ receives $T_{i+6}^{3}$ and $S_{i+7}^{3}$. Therefore,

$$
\begin{aligned}
& T^{6}=(511,511,511,511,511,511,511,511,511) \\
& S^{6}=(510,509,507,503,495,479,447,383,255) .
\end{aligned}
$$

Indeed, $D=d_{0}+d_{1}+\cdots+d_{8}=1+2+\cdots+256=511$.

\section{ACKNOWLEDGMENTS}

Jehoshua Bruck was supported in part by the National Science Foundation Young Investigator Award CCR-9457811; by the Sloan Research Fellowship; by a grant from the IBM Almaden Research Center, San Jose, California; and by a grant from the AT\&T Foundation.

\section{REFERENCES}

[1] A. Bagchi, E. f. Schmeichel, and S.L. Hakimi, "Sequential information dissemination by packets," Networks, vol. 22, no. 4, pp. 317333, July 1992.

[2] V. Bala, J. Bruck, R. Bryant, R. Cypher, P. deJong, P. Elustondo, D. Frye, A. Ho, C.T. Ho, G. Irwin, S. Kipnis, R. Lawrence, and M. Snir, "The IBM external user interface for scalable parallel systems," Parallel Computing, vol. 20, no. 4, pp. 445-462, Apr. 1994. 
[3] V. Bala, J. Bruck, R. Cypher, P. Elustondo, A. Ho, C.T. Ho, S. Kipnis, and $\mathrm{M}$. Snir, "CCL: A portable and tunable collective communication library for scalable parallel computers," Eighth Int'l Parallel Processing Symp., IEEE, pp. 835-844, Apr. 1994.

[4] M. Barnett, R. Littlefield, D.G. Payne, and R. van de Geijn, "Global combine on mesh architectures with wormhole routing," Seventh Int'l Parallel Processing Symp., IEEE, Apr. 1993.

[5] A. Bar-Noy and S. Kipnis, "Designing broadcasting algorithms in the postal model for message-passing systems," Math. Systems Theory, vol. 27 , no. 5 , pp. $431-452,1994$.

[6] A. Bar-Noy and S. Kipnis, "Multiple message broadcasting in the postal model, Proc. Seventh Int'l Parallel Processing Symp., IEEE, Apr. 1993.

[7] A. Bar-Noy and S. Kipnis, "Broadcasting multiple messages in simultaneous send/receive systems, Fifth Symp. Parallel and Distributed, Processing, IEEE, pp. 344-347, Dec. 1993.

[8] A. Bar-Noy, S. Kipnis, and B. Schieber, "An optimal algorithm for computing census functions in message-passing systems," Parallel Processing Letters, vol. 3, no. 1, pp. 19-23, Mar. 1993.

[9] A. Bar-Noy, S. Kipnis, and B. Schieber, "Optimal computation of census functions in the postal model," to appear in Discrete Applied Math.

[10] J. Bruck, L. de Coster, N. Dewulf, C.T. Ho, and R. Lauwereins, "On the design and implementation of broadcast and global combine operations using the postal model," Sixth Symp. Parallel and Distributed Processing, IEEE, pp. 594-602, Oct. 1994.

[11] J. Bruck, R. Cypher, and C.T. Ho, "Multiple message broadcasting with generalized Fibonacci trees," Fourth Symp. Parallel and Distributed Processing, IEEE, pp. 424-431, Dec. 1992.

[12] J. Bruck, C.T. Ho, S. Kipnis, and D. Weathersby, "Efficient algorithms for all-to-all communications in multiport message-passing systems," Sixth Ann. Symp. Parallel Algorithms and Architectures, ACM, pp. 298-309, June 1994.

[13] J. Bruck, C.T. Ho, "Efficient global combine operations in multiport message-passing systems," Parallel Proc. Letters, vol. 3, no. 4 pp. 335-346, Dec. 1993.

[14] I. Cidon and I. Gopal, "PARIS: An approach to integrated high-speed private networks," Int'l J. Digital and Analog Cabled Systems, vol. 1, no. 2, pp. 77-85, Apr.-June 1988.

[15] D. Clark, B. Davie, D. Farber, I. Gopal, B. Kadaba, D. Sincoskie, J. Smith, and D. Tennenhouse, "The AURORA gigabit testbed," Computer Networks and ISDN, 1991
[16] D. Culler, A.C. Dusseau, R.P. Martin, and K.E. Schauser, "Fast paralle sorting under LogP: From theory to practice," Workshop on Portability and Performance for Parallel Processing, Southampton, England, 1993.

[17] D. Culler, R. Karp, D. Patterson, A. Sahay, K.E. Schauser, E. Santos, R. Subramonian, and T. von Eicken, "LogP: Towards a realistic model of parallel computation," Fourth Symp. Principles and Practices Parallel Programming, SIGPLAN '93, ACM, May 1993.

[18] J. Dongarra et al., "Document for a standard message-passing interface," Message Passing Interface Forum, Univ. of Tennessee, Tech. Report CS-93-214, Nov. 1993.

[19] Express 3.0 Introductory Guide. Parasoft Corporation, 1990.

[20] G. Fox, M. Johnson, G. Lyzenga, S. Otto, J. Salmon, and D. Walker, Solving Problems on Concurrent Processors, Vol. I: General Techniques and Regular Problems. Englewood Cliffs, N.J.: Prentice Hall 1988.

[21] S.M. Hedetniemi, S.T. Hedetniemi, and A.L. Liestman, "A survey of gossiping and broadcasting in communication networks," Networks, vol. 18, no. 4, pp. 319-349, 1988.

[22] S.L. Johnsson and C.T. Ho, "Spanning graphs for optimum broadcasting and personalized communication in hypercubes," IEEE Trans. Computers, vol. 38, no. 9, pp. 1,249-1,268, Sept. 1989.

[23] R. Karp, A. Sahay, E. Santos, and K.E. Schauser, "Optimal broadcas and summation in the LogP model," Proc. Fifth Ann. Symp. Parallel Algorithms and Architectures, ACM, June 1993.

[24] C.E. Leiserson, Z.S. Abuhamdeh, D.C. Douglas, C.R. Feynman, M.N. Ganmuki, J.V. Hill, W.D. Hillis, B.C. Kuszmaul, M.A. St. Pierre, D.S. Wells, M.C. Wong, S.-W. Yang, and R. Zak, "The network architecture of the connection machine CM-5," Proc. Fourth Ann. Symp. Parallel Algorithms and Architectures, ACM, pp. 272-285, June 1992.

[25] J.F. Palmer, "The NCUBE family of parallel supercomputers," Int'l Conf. Computer Design, IEEE, 1986

[26] Q.F. Stout and B. Wagar, "Intensive hypercube communication: Prearranged communication in link-bound machines," J. Parallel and Distributed Computing, vol. 10, pp. 167-181, 1990.

[27] R.A. van de Geijn, "Efficient global combine operations," Sixth Distributed Memory Computing Conf., IEEE, Apr. 1991.

[28] C.B. Stunkel, D. S. Shea, B. Abali, M M. Denneau, P.H. Hochschild, D.J. Joseph, B.J. Nathanson, M. Tsao, and P.R. Varker, "Architectures and implementation of vulcan," Eighth Int'l Parallel Processing Symp., IEEE, pp. 268-274, Apr. 1994. 